\title{
Flexible joints in structural and multibody dynamics
}

\section{O. A. Bauchau and S. Han}

\author{
University of Michigan - Shanghai Jiao Tong University Joint Institute, Shanghai, 200240, China \\ Correspondence to: O. A. Bauchau (olivier.bauchau@sjtu.edu.cn)
}

Received: 29 October 2012 - Revised: 2 January 2013 - Accepted: 24 January 2013 - Published: 13 February 2013

\begin{abstract}
Flexible joints, sometimes called bushing elements or force elements, are found in all structural and multibody dynamics codes. In their simplest form, flexible joints simply consist of sets of three linear and three torsional springs placed between two nodes of the model. For infinitesimal deformations, the selection of the lumped spring constants is an easy task, which can be based on a numerical simulation of the joint or on experimental measurements. If the joint undergoes finite deformations, identification of its stiffness characteristics is not so simple, specially if the joint is itself a complex system. When finite deformations occur, the definition of deformation measures becomes a critical issue. This paper proposes a family of tensorial deformation measures suitable for elastic bodies of finite dimension. These families are generated by two parameters that can be used to modify the constitutive behavior of the joint, while maintaining the tensorial nature of the deformation measures. Numerical results demonstrate the objectivity of the deformations measures, a feature that is not shared by the deformations measures presently used in the literature. The impact of the choice of the two parameters on the constitutive behavior of the flexible joint is also investigated.
\end{abstract}

\section{Introduction}

Flexible joints, sometimes called bushing elements or force elements, are found in all multibody dynamics codes. In their simplest form, flexible joints simply consist of sets of three linear and three torsional springs placed between two nodes of a multibody system. For infinitesimal deformations, the selection of the lumped spring constants is an easy task, which can be based on a numerical simulation of the joint or on experimental measurements.

If the joint undergoes finite deformations, identification of its stiffness characteristics is not so simple, specially if the joint is itself a complex system. When finite deformations occur, the definition of the deformation measures becomes a critical issue. Indeed, for finite deformation, the observed nonlinear behavior of materials is partly due to material characteristics, and partly due to kinematics.

For instance, Anand $(1979,1986)$ has shown that the classical strain energy function for infinitesimal isotropic elasticity is in good agreement with experiment for a wide class of materials for moderately large deformations, provided the infinitesimal strain measure used in the strain energy function is replaced by the Hencky or logarithmic measure of finite strain. This means that the behavior of materials for moderate deformations can be captured accurately using linear constitutive laws, provided that the infinitesimal strain measures are replaced by finite deformation measures that are nonlinear functions of displacement.

These nonlinear deformation measures capture the observed nonlinear behavior associated with the nonlinear kinematics of the problem. Degener et al. (1988) also reported similar findings for the torsional behavior of beams subjected to large axial elongation.

Much attention has been devoted to the problem of synthesizing accurate constitutive properties for the modeling of flexible bushings presenting complex, time-dependent rheological behavior, Ledesma et al. (1996); Kadlowec et al. (2003). It is worth stressing, however, that the literature seldom addresses three-dimensional joint deformations.

Much like multibody codes, most FE codes also support the modeling of lumped structural elements. While linear analysis is easily implemented, problems are encountered when dealing with finite displacements and rotations, as pointed out by Masarati and Morandini (2010). Structural analysis codes, either specifically intended for multibody dynamics analysis, like MSC/ADAMS, or for nonlinear FEA 
with multibody capabilities, like Abaqus/Standard, allow arbitrarily large absolute displacements and rotations of the nodes and correctly describe their rigid-body motion. When lumped deformable joints are used, relative displacements and rotations are often required to remain moderate, although not necessarily infinitesimal.

Such restrictions occur when using the FIELD element of MSC/ADAMS, a linear element that implements an orthotropic torsional spring based on a constant, orthotropic constitutive matrix. Similarly, the JOINTC element implemented in Abaqus/Standard describes the interaction between two nodes when the second node can "displace and rotate slightly with respect to the first node", because its formulation is based on an approximate relative rotation measure.

The formulations and implementations of flexible joints available in research and commercial codes do not appear to allow arbitrarily large relative displacements and rotations. Moreover, in many cases, the ordering sequence of the nodes connected to the joint matters, because the behavior of the flexible joint is biased towards one of the nodes. This problem is known to experienced analysts using these codes. To the authors' knowledge, these facts are rarely acknowledged in the literature. It appears that little effort has been devoted to the elimination of these shortcomings from the formulations found in research and commercially available codes, although the predictions of these codes might be unexpected.

This paper presents families of finite deformation measures that can be used to characterize the deformation of flexible joints. These deformation measures are closely related to the tensorial parameterization motion developed by Bauchau and $\mathrm{Li}$ (2011); Bauchau (2011). Because they are of a tensorial nature, these deformation measures are intrinsic and invariant. Numerical examples demonstrate the invariance of the formulation and the ability to tailor the joint's constitutive behavior in the nonlinear range. Section 2 describes the configuration of the flexible joint. Section 3 presents the proposed deformation measures, which are derived from invariance considerations.

\section{Flexible joint configuration}

\subsection{Kinematics of the flexible joint}

Figure 1 shows inertial frame $\mathcal{F}^{I}=\left[\mathbf{O}, \mathcal{I}=\left(\bar{l}_{1}, \bar{l}_{2}, \bar{l}_{3}\right)\right]$ and a flexible joint in its reference and deformed configurations. It consists of a three-dimensional elastic body of finite dimension and of two rigid bodies, called handle $k$ and handle $\ell$, that are rigidly connected to the elastic body. In the reference configuration, the configuration of the handles is defined by frame $\mathcal{F}_{0}=\left[\mathbf{B}, \mathcal{B}_{0}=\left(\bar{b}_{01}, \bar{b}_{02}, \bar{b}_{03}\right)\right]$, where $\mathcal{B}_{0}$ forms an orthonormal basis. The geometric location of points $\mathbf{K}$ and $\mathbf{L}$, which are material points of handles $k$ and $\ell$, respectively, coincides with that of point $\mathbf{B}$. The motion tensor that brings frame $\mathcal{F}^{I}$ to frame $\mathcal{F}_{0}$ is denoted $\underline{\underline{C}}_{0}\left(\underline{u}_{0}, \underline{\underline{R}}_{0}\right)$, where $\underline{u}_{0}$ is the

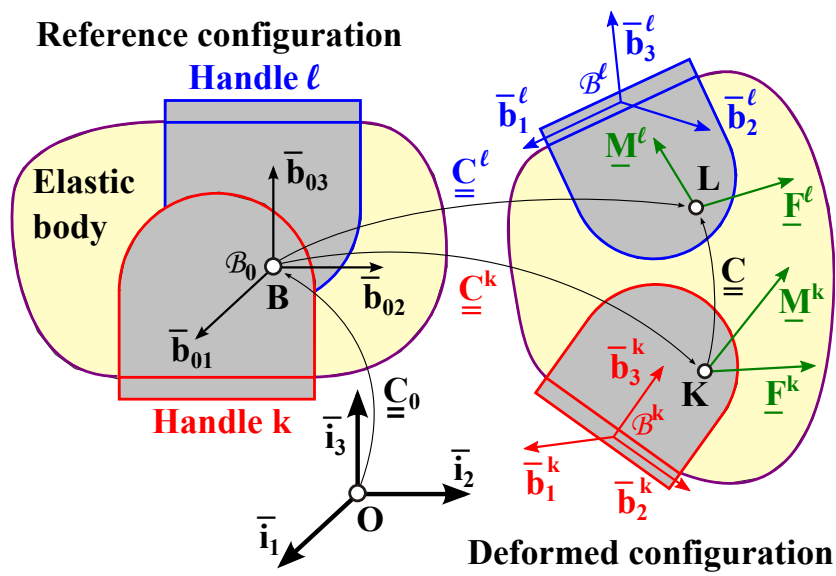

Figure 1. Configuration of the flexible joint.

position vector of point $\mathbf{B}$ with respect to point $\mathbf{O}$ and $\underline{R}_{0}$ is the rotation tensor that brings basis $\mathcal{I}$ to basis $\mathcal{B}_{0}$.

In the deformed configuration, the two handles move to new positions and the elastic body deforms. Materials points $\mathbf{K}$ and $\mathbf{L}$ are now at distinct locations. The configurations of the two handles are now distinct and are represented by two distinct frames, $\mathcal{F}^{k}=\left[\mathbf{K}, \mathcal{B}^{k}=\left(\bar{b}_{1}^{k}, \bar{b}_{2}^{k}, \bar{b}_{3}^{k}\right)\right]$ and $\mathcal{F}^{\ell}=$ $\left[\mathbf{L}, \mathcal{B}^{\ell}=\left(\bar{b}_{1}^{\ell}, \bar{b}_{2}^{\ell}, \bar{b}_{3}^{\ell}\right)\right]$, respectively. Motion tensors $\underline{\mathcal{C}}^{k}$ and $\underline{\mathcal{C}}^{\ell}$ bring frame $\mathcal{F}_{0}$ to frames $\mathcal{F}^{k}$ and $\mathcal{F}^{\ell}$, respectively. The displacement vectors from point $\mathbf{B}$ to points $\mathbf{K}$ and $\mathbf{L}$, are denoted $\underline{u}^{k}$ and $\underline{u}^{\ell}$, respectively, and rotation tensors $\underline{\underline{R}}^{k}$ and $\underline{\underline{R}}^{\ell}$ bring basis $\mathcal{B}_{0}$ to bases $\mathcal{B}^{k}$ and $\mathcal{B}^{\ell}$, respectively.

Relative motion tensor $\underline{\underline{C}}$ brings frame $\mathcal{F}^{k}$ to frame $\mathcal{F}^{\ell}$ and provides an intrinsic representation of the motion of handle $\ell$ with respect to handle $k$. The relative displacement vector of point $\mathbf{L}$ with respect to point $\mathbf{K}$ is denoted $\underline{u}=\underline{u}^{\ell}-\underline{u}^{k}$ and $\underline{R}=\left(\underline{R}^{\ell} \underline{R}_{0}\right)\left(\underline{R}^{k} \underline{R}_{0}\right)^{T}$ is the relative rotation tensor of basis $\mathcal{B}^{\ell}$ with respect to basis $\mathcal{B}^{k}$.

The motion tensors that bring frame $\mathcal{F}^{I}$ to frames $\mathcal{F}^{k}$ and $\mathcal{F}^{\ell}$, denoted $\stackrel{\mathbb{C}}{=}^{k}$ and $\stackrel{\mathbb{C}}{=}^{\ell}$, respectively can be expressed as

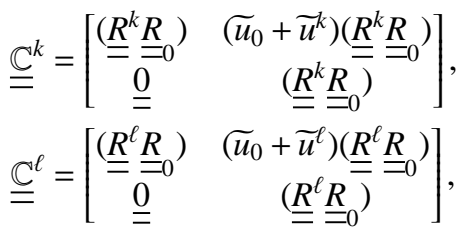

respectively. The relative motion tensor then becomes

$\underline{\underline{C}}=\underline{\mathbb{C}}^{\ell} \underline{\mathbb{C}}^{k-1}$

The components of relative motion tensor resolved in frames $\mathcal{F}^{k}$ and $\mathcal{F}^{\ell}$ are identical,

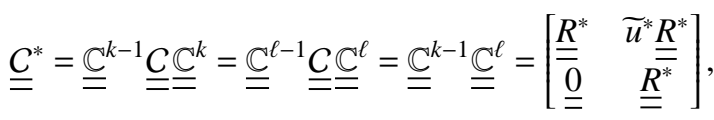


where $\left.\quad \underline{R}^{*}=\left(\underline{\underline{R}}^{k} \underline{\underline{R}}\right)^{T} \underline{\underline{R}}_{\underline{\underline{R}}}^{\underline{\underline{R}}} \underline{\underline{R}}_{0}\right)=\left(\underline{\underline{R}}^{\ell} \underline{\underline{R}}_{0}\right)^{T} \underline{\underline{R}}\left(\underline{\underline{R}}^{\ell} \underline{\underline{R}}_{0}\right)=\left(\underline{\underline{R}}^{k} \underline{\underline{R}}_{0}\right)^{T}$ $\left(\underline{R}^{\ell} \underline{R}_{0}\right)$ are the components of the relative rotation tensor resolved in bases $\mathcal{B}^{k}$ or $\mathcal{B}^{\ell}$ and $\underline{u}^{*}=\left(\underline{R}^{k} \underline{R}_{0}\right)^{T}\left(\underline{u}^{\ell}-\underline{u}^{k}\right)$.

\subsection{Applied loading}

The deformation of the flexible joint stems from the applied forces and moments depicted in Fig. 1. At point $\mathbf{K}$, the applied force and moment vectors are denoted $\underline{F}^{k}$ and $\underline{M}^{k}$, respectively; the corresponding quantities applied at point $\mathbf{L}$ are denoted $\underline{F}^{\ell}$ and $\underline{M}^{\ell}$, respectively. The loading applied to the flexible joint is characterized by arrays $\underline{\mathcal{A}}^{k}$ and $\underline{\mathcal{A}}^{\ell}$ that correspond to a translation of these loads to point $\mathbf{O}$,

$\underline{\mathcal{A}}^{k}=\underline{\mathcal{T}}^{k-T}\left\{\underline{F}^{k} \underline{M}^{k}\right\}=\left\{\underline{M}^{k}+\left({\underline{\bar{u}_{0}}}^{k}+\widetilde{u}^{k}\right) \underline{F}^{k}\right\}$,

$\underline{\mathcal{A}}^{\ell}=\underline{\mathcal{T}}^{\ell-T}\left\{\underline{F}^{\ell}\right\}=\left\{\begin{array}{c}\underline{F}^{\ell} \\ \underline{M}^{\ell}+\left(\widetilde{u}_{0}+\widetilde{u}^{\ell}\right) \underline{F}^{\ell}\end{array}\right\}$,

where $\mathcal{T}^{k}$ and $\mathcal{T}^{\ell}$ are the translation tensors from point $\mathbf{O}$ to points $\overline{\overline{\mathbf{K}}}$ and $\overline{\mathbf{L}, \text { respectively, defined as }}$

$\underline{\mathcal{T}}^{k}=\left[\begin{array}{cc}\underline{\underline{I}} & \left(\widetilde{u}_{0}+\widetilde{u}^{k}\right) \\ \underline{\underline{0}} & \underline{\underline{I}}\end{array}\right]$

$\underline{\mathcal{T}^{\ell}}=\left[\begin{array}{cc}\underline{\underline{I}} & \left(\widetilde{u}_{0}+\widetilde{u}^{\ell}\right) \\ \underline{\underline{0}} & \underline{\underline{I}}\end{array}\right]$,

respectively. Because these loads are both applied at point $\mathbf{O}$, the equilibrium condition resulting from Newton's third law simply states

$\underline{\mathcal{A}}^{k}+\underline{\mathcal{A}}^{\ell}=\underline{0}$.

Note the parallel between vectors $\mathcal{A}^{k}$ and $\mathcal{A}^{\ell}$ and the second Piola-Kirchhoff stress tensor (Malvern, 1969). Indeed, they represent the true loads applied to handle $k$ and $\ell$, respectively, in their deformed configurations, but translated to reference point $\mathbf{O}$. Although expressing equilibrium of the system in its deformed configuration, Eq. (6) is a linear function of the loads. The joint is assumed to be massless, i.e., inertial forces associated with its motion are neglected.

\section{Deformation measures}

The differential work done by the applied load will be evaluated in Sect. 3.1, and the nature of deformation measures is further discussed in Sect. 3.2. Section 3.3 then provides invariant deformation measures based on the tensorial parameterization of motion.

\subsection{Differential work}

The differential work, $\mathrm{d} W$, done by the forces applied to the joint is $\mathrm{d} W=\underline{F}^{k T} \mathrm{~d} \underline{u}^{k}+\underline{M}^{k T} \mathrm{~d} \psi^{k}+\underline{F}^{\ell T} \mathrm{~d} \underline{u}^{\ell}+\underline{M}^{\ell T} \mathrm{~d} \psi^{\ell}$, where $\mathrm{d} \underline{u}^{k}$ and $\mathrm{d} \underline{u}^{\ell}$ are the differential displacements of point $\overline{\mathbf{K}}$ and $\mathbf{L}$, respectively, and $\underline{\mathrm{d} \psi^{k}}=\operatorname{axial}\left(\mathrm{d} \underline{R}^{k} \underline{R}^{k T}\right)$ and $\underline{\mathrm{d} \psi^{\ell}}=$ $\operatorname{axial}\left(\mathrm{d} \underline{R}^{\ell} \underline{R}^{\ell T}\right)$ the differential rotations of handles $k$ and $\bar{\ell}$, respectively. Recasting this expression in a matrix form leads to

$$
\begin{aligned}
\mathrm{d} W= & \left\{\underline{\mathrm{d}}^{k T}, \underline{\mathrm{d} \psi^{k T}}\right\} \underline{\mathcal{T}}^{k T} \underline{\mathcal{T}}^{k-T}\left\{\underline{F}^{k}\right\} \\
& +\left\{\mathrm{d} \underline{\underline{M}}^{\ell T}, \underline{\mathrm{d} \psi^{\ell T}}\right\} \underline{\mathcal{T}^{\ell T}} \stackrel{\mathcal{T}^{\ell-T}}{=}\left\{\underline{F}^{\ell}\right\} \\
= & \underline{\mathrm{d}}^{k T} \underline{\mathcal{A}}^{k}+\underline{\mathrm{d}}^{\ell T} \underline{\mathcal{A}}^{\ell},
\end{aligned}
$$

where the last equality follows from Eq. (4). The differential motions of handles $k$ and $\ell$ are defined as $\mathrm{d} \mathcal{U}^{k}=$ $\mathcal{A} x i a l\left(\mathrm{~d} \stackrel{\mathbb{C}}{k}^{k} \mathbb{C}^{k-1}\right)$ and $\underline{\mathrm{d} \mathcal{U}^{\ell}}=\mathcal{A x i a l}\left(\mathrm{d} \mathbb{C}_{=}^{\ell} \mathbb{C}^{\ell-1}\right)$, respectively,

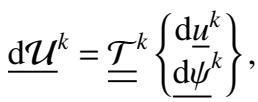

$\underline{\mathrm{d} \mathcal{U}^{\ell}}=\underline{\mathcal{T}^{\ell}}\left\{\begin{array}{l}\mathrm{d} \underline{u}^{\ell} \\ \mathrm{d} \psi^{\ell}\end{array}\right\}$,

respectively, where operator $\mathcal{A x i a l}(\cdot)$ is defined by Eq. (A3). Finally, introducing Eq. (6), the differential work becomes

$\mathrm{d} W=\underline{\mathcal{A}}^{\ell T}\left(\underline{\mathrm{d} \mathcal{U}^{\ell}}-\underline{\mathrm{d} \mathcal{U}^{k}}\right)$.

The term in parenthesis represents the differential relative motion, $\underline{\mathrm{d} \mathcal{U}}=\underline{\mathrm{d} \mathcal{U}^{\ell}}-\underline{\mathrm{d} \mathcal{U}}{ }^{k}$, where $\underline{\mathrm{d} \mathcal{U}}=\mathcal{A} \operatorname{xial}\left(\mathrm{d} \underline{C} \underline{C}^{-1}\right)$. As expected, the differential work depends on the $\overline{\overline{d i f f}}$ erential relative motion of the two handles only. The differential work can now be written as $\mathrm{d} W=\underline{\mathcal{A}}^{\ell T} \underline{\mathrm{d} \mathcal{U}}=\underline{\mathcal{A}}^{k T}(-\underline{\mathrm{d} \mathcal{U}})$, where the second equality follows from the equilibrium equation, Eq. (6). This statement simply implies that the differential relative motion of handle $\ell$ with respect to handle $k$, denoted $\mathrm{d} \mathcal{U}$ is of opposite sign of that of handle $k$ with respect to handle $\ell$, as expected. In summary, the differential work is written

$\mathrm{d} W=\underline{\mathcal{A}}{ }^{T} \underline{\mathrm{d}}$,

where by convention, $\underline{\mathcal{A}}=\underline{\mathcal{A}^{\ell}}$ and $\underline{\mathrm{d} \mathcal{U}}=\underline{\mathrm{d} \mathcal{U}^{\ell}}-\underline{\mathrm{d} \mathcal{U}^{k}}$ the differential relative motion of handle $\ell$ with respect to handle $k$.

Let $\underline{\mathcal{E}}$ be a set of six generalized coordinates that define the relative motion tensor uniquely, i.e., a one-to-one mapping is assumed to exist between these generalized coordinates and the relative motion tensor. It follows that a one-to-one mapping must exist between the relative motion tensor and increments of the generalized coordinates

$\underline{\mathrm{d} \mathcal{U}}=\underline{\underline{H}}(\underline{\mathcal{E}}) \mathrm{d} \underline{\mathcal{E}}$,

where tensor $\underline{\underline{H}}(\underline{\mathcal{E}})$ is the Jacobian or tangent tensor of the coordinate transformation. The differential work done by the forces applied to the joint, Eq. (8), now becomes 
$\mathrm{d} W=\underline{\mathcal{A}}^{T} \underline{\mathcal{H}}(\underline{\mathcal{E}}) \mathrm{d} \underline{\mathcal{E}}=\underline{\mathcal{L}}^{T} \mathrm{~d} \underline{\mathcal{E}}$, where the generalized forces associated with the generalized coordinates are defined as

$\underline{\mathcal{L}}=\underline{\underline{\mathcal{H}}}^{T}(\underline{\mathcal{E}}) \underline{\mathcal{A}}$.

It is assumed that the flexible joint is made of an elastic material (Bauchau and Craig, 2009), which implies that the generalized forces can be derived from a potential, the strain energy of the joint, denoted $A$,

$\mathcal{L}=\frac{\partial A(\underline{\mathcal{E}})}{\partial \underline{\mathcal{E}}}$.

The differential work now becomes

$\mathrm{d} W=\mathrm{d} \underline{\mathcal{E}}^{T} \frac{\partial A(\underline{\mathcal{E}})}{\partial \underline{\mathcal{E}}}=\mathrm{d}(A)$,

and can be expressed as the differential of a scalar function, the strain energy.

\subsection{The deformation measures}

In the previous section, quantities $\underline{\mathcal{E}}$ were defined as "a set of generalized coordinates that uniquely define the relative motion tensor," but were otherwise left undefined. This implies that these generalized coordinates form a parameterization of the relative motion tensor, i.e., $\underline{\underline{C}}=\underline{\underline{C}}(\underline{\mathcal{E}})$. Because the strain energy of the flexible joint can be expressed in terms of these generalized coordinates they are, in fact, deformation measures for the flexible joint. Consequently, any parameterization of the relative motion tensor provides deformation measures for the flexible joint.

The following notation is introduced

$\underline{\mathcal{E}}=\left\{\begin{array}{l}\underline{\varepsilon} \\ \underline{\kappa}\end{array}\right\}$.

The first three components of this array form the stretch vector, denoted $\underline{\varepsilon}$, and the last three the wryness vector, denoted $\underline{\kappa}$. Although any parameterization of the relative motion tensor provides adequate deformation measures for the flexible joint, these deformation measures should be invariant with respect to the choice of coordinate system. This implies that the stretch and wryness vectors should be first-order tensors, and hence, the deformation measure should itself be a firstorder tensor.

While the parameterization of rotation has received wide attention (Kane, 1968; Argyris, 1982; Shuster, 1993; Ibrahimbegović, 1997; Bauchau and Trainelli, 2003), much less emphasis has been placed on that of motion (Angeles, 1993; Borri et al., 2000; Merlini and Morandini, 2004; Pennestrì and Stefanelli, 2007). Bauchau and Choi (2003) and Bauchau and Li (2011) have proposed the vectorial parameterization of motion, which consists of a motion parameter vector, $\underline{\mathcal{E}}$, that parameterizes the motion tensor, $\underline{\underline{C}}=$
$\underline{\underline{C}}(\underline{\mathcal{E}})$. Bauchau and $\mathrm{Li}$ (2011) and Bauchau (2011) have studied the parameterization of motion, with special emphasis on its tensorial nature. They presented a formal proof that motion parameters vectors are first-order tensors if and only if they are parallel to the eigenvectors of the motion tensor associated with its unit eigenvalues. Furthermore, the tangent tensor is also a second-order tensor for motion parameters vectors.

Deformation measures should be "objective" or "frameindifferent", and Malvern (1969) gives a precise definition of this concept, which implies that deformation measures should be expressed in terms of tensorial quantities and be invariant under the superposition of a rigid body motion. The relative motion tensor remains invariant under the superposition of a rigid body motion and hence, deformation measures that are functions of the relative motion tensor only will share this property. The previous paragraphs have underlined the fact that the deformation measures should be of a tensorial nature. If this latter property is achieved, the resulting deformation measures will be objective.

In summary, the desired invariance and objectivity of the deformation measure are achieved if and only if this deformation measure is selected to be the vectorial parameterization of motion, which further implies that the deformation measure is parallel to the eigenvector of the motion tensor associated with its unit eigenvalue.

\subsection{Explicit expression of the deformation measures}

The proposed deformation measures are parallel to the eigenvector of the relative motion tensor associated with its unit eigenvalue. Because this eigenvalue has a multiplicity of two (Bauchau, 2011), two linearly independent eigenvectors exist, which will be selected as

$\underline{\mathcal{N}}_{1}^{\dagger}=\{\underline{p} \underline{\underline{p}}\}, \quad$ and $\quad \underline{\overline{\mathcal{N}}}_{2}^{\dagger}=\left\{\begin{array}{c}\underline{\underline{H}}^{-1} \underline{u} \\ \underline{p}\end{array}\right\}$,

where $p$ is the Wiener-Milenković rotation parameter vector (Wiener, 1962; Milenković, 1982; Bauchau, 2011) and $\underline{H}$ the tangent tensor for this parameterization. The second eigenvector is easily recognized as the Wiener-Milenković motion parameter vector (Bauchau, 2011), i.e.,

$\underline{\mathcal{N}}_{2}^{\dagger}=\underline{\mathcal{P}}=\{\underline{q} \underline{p}\}$.

The following notation is introduced: $p=\|p\|=4 \tan \phi / 4$, $\varrho=p^{T} q=p p^{\prime} d$, where $d=\bar{n}^{T} \underline{u}$ is the intrinsic displacement,

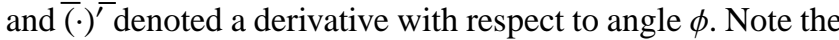
following limit behaviors: (1) $\lim _{\phi \rightarrow 0} p=\phi$, (2) $\lim _{\phi \rightarrow 0} \underline{\underline{H}}^{-1}=$ $\underline{I}$ and hence, $\lim _{\phi \rightarrow 0} \underline{q}=\underline{u}$, and (3) $\lim _{d \rightarrow 0} \varrho=0$;

The most general measure of deformation is a linear combination of vectors $\underline{\mathcal{N}}_{1}^{\dagger}$ and $\overline{\mathcal{N}}_{2}^{\dagger}$ defined by Eq. (14), which 
will be written as

$\underline{\mathcal{E}}=\left[\begin{array}{ll}\mu \underline{\underline{I}} & \lambda \underline{\bar{I}} \\ \underline{\underline{0}} & \mu \underline{\underline{I}}\end{array}\right] \underline{\mathcal{P}}=\underline{\underline{Z}}(\lambda, \mu) \underline{\mathcal{P}}$,

where matrix $\mathcal{Z}$ is defined in Eq. (A1), and $\lambda$ and $\mu$ are arbitrary scalars. The stretch and wryness vectors now become $\underline{\varepsilon}=\mu q+\lambda p$ and $\underline{\kappa}=\mu p$, respectively. Mozzi-Chasles' theorem implies that a geñeral motion can be defined in terms of the direction of axis of the motion and in terms of two scalar parameters, $\phi$, the magnitude of the relative rotation, and $d$, the intrinsic relative displacement. Equivalently, scalar functions $\lambda$ and $\mu$ can be expressed in terms of $\varrho=p^{T} q$ and $p=\sqrt{\underline{p}^{T} \underline{p}}$, i.e.,

$\lambda=\lambda(\varrho, p), \quad \mu=\mu(\varrho, p)$.

Although functions $\lambda$ and $\mu$ are arbitrary, their limit behavior for $\varrho$ and $p \rightarrow 0$ can be obtained based on physical arguments. The wryness vector is written as $\underline{\kappa}=\mu p \bar{n}$; since $\mu p$ must be an odd function of $\phi$ (or $p$ ), it implies that $\mu$ is an even function of the same variable. For small values of $\phi$ (or $p$ ), the wryness vector should be equal to the infinitesimal rotation vector, i.e., $\lim _{p \rightarrow 0} \underline{\kappa}=\phi \bar{n}$. Introducing the expression for the wryness vector yields $\lim _{\phi \rightarrow 0} 4 \mu \tan (\phi / 4) \bar{n}=$ $\lim _{\phi \rightarrow 0} \mu \phi \bar{n}=\phi \bar{n}$, which implies

$\lim _{\phi \rightarrow 0} \mu=1$.

Similarly, for small rotation angles, the stretch vector should equal the displacement vector, i.e., $\lim _{\phi \rightarrow 0} \varepsilon=u$. Introducing the expression for the stretch vector yields $\lim _{\phi \rightarrow 0}\left(\mu \underline{\underline{H}}^{-1} \underline{u}+\right.$ $4 \lambda \tan (\phi / 4) \bar{n})=\underline{u}$. Given the limit behaviors of $\mu$ and $\underline{H}^{-1}$, the stretch vector does indeed converge to the displacement vector. Finally, if the relative motion is planar, the intrinsic displacement vanishes and the stretch vector should be in the plane normal to unit vector $\bar{n}$, i.e., $\lim _{d \rightarrow 0} \bar{n}^{T} \underline{\varepsilon}=0$. Using the expression for the stretch vector then leads to $\lim _{d \rightarrow 0}\left(\mu p^{\prime} d+\right.$ $4 \lambda \tan \phi / 4)=0$, which implies

$\lim _{d \rightarrow 0} \lambda=0$.

In summary, Eq. (16) defines the proposed deformation measures for flexible joints. These equations are, in fact, the nonlinear deformation-displacement relationships of the problem. They are not fully determined because two scalar functions, $\lambda$ and $\mu$, appear in their definition. These two functions must satisfy the limit behavior discussed earlier but are otherwise arbitrary. Specific choices of these two scalar functions result in different families of deformation measures.

\section{Formulation of flexible joints}

The strain energy of the flexible joint is assumed to be a quadratic function of the deformation measures $\underline{\mathcal{E}}^{*}$,

$A\left(\underline{\mathcal{E}}^{*}\right)=\frac{1}{2} \underline{\mathcal{E}}^{* T} \underline{\underline{D}}^{*} \underline{\mathcal{E}}^{*}$, where $\mathcal{D}^{*}$ are the components of the stiffness matrix of the flexible joint resolved in the material frame, which are assumed to be given constants in this frame.

\subsection{Elastic forces in the flexible joint}

The components of relative motion tensor resolved in the material frame are given by Eq. (3) and its variation becomes

$$
\begin{aligned}
\widetilde{\delta \mathcal{U}}^{*} & =\delta \underline{\underline{C}}^{*} \underline{\underline{C}}^{*-1}=\left(\underline{\delta \mathbb{C}^{k-1}} \underline{\mathbb{C}}^{\ell}+\underline{\mathbb{C}}^{k-1} \delta \underline{\mathbb{C}}^{\ell}\right) \underline{\underline{C}}^{\ell-1} \stackrel{\mathbb{C}}{ }^{k} \\
& =\underline{\mathbb{C}}^{k-1}\left(\widetilde{\delta \mathcal{U}}^{\ell}-\widetilde{\delta \mathcal{U}}^{k}\right) \underline{\mathbb{C}}^{k},
\end{aligned}
$$

where the virtual motion vectors, $\delta \mathcal{U}^{k}$ and $\delta \mathcal{U}^{\ell}$, are defined in Eqs. (7a) and (7b), respectively. It now follows that

$\underline{\delta \mathcal{U}}^{*}=\underline{\mathbb{C}}^{k-1}\left(\underline{\delta \mathcal{U}}^{\ell}-\underline{\delta \mathcal{U}^{k}}\right)$

The virtual motion vector is related to the virtual changes in the motion parameter vector (Bauchau, 2011) by means of the tangent tensor, $\underline{\mathcal{H}}$, as $\underline{\delta \mathcal{U}}{ }^{*}=\underline{\mathcal{H}}\left(\underline{\mathcal{P}}^{*}\right) \delta \underline{\mathcal{P}}^{*}$. It then follows that $\delta \underline{\mathcal{P}}^{*}=\underline{\mathcal{H}}^{-1}\left(\underline{\mathcal{P}}^{*}\right) \underline{\overline{\delta \mathcal{U}}}{ }^{*}=\underline{\mathcal{H}}^{-1}\left(\underline{\overline{\mathcal{P}^{k}}}\right) \underline{\mathbb{C}}^{k-1}\left(\underline{\delta \mathcal{U}}{ }^{\ell}-\underline{\delta \mathcal{U}}{ }^{k}\right)$, where the second equality was obtained with the help of Eq. (21). The motion tensor affords the following multiplicative decomposition (Bauchau, 2011), $\underline{\underline{C}}^{*}\left(\underline{\mathcal{P}}^{*}\right)=\underline{\underline{\mathcal{H}}}\left(\underline{\mathcal{P}}^{*}\right) \underline{\mathcal{H}}^{*-1}\left(\underline{\mathcal{P}}^{*}\right)$ Equation (3) then implies $\underline{\underline{C}}^{\ell} \underline{\mathcal{H}}^{*}=\underline{\mathbb{C}}^{k} \underline{\underline{H}}$, and tensor $\underline{\underline{W}}$ is defined as

$\underline{\underline{W}}=\left(\underline{\mathbb{C}}^{k} \underline{\underline{\mathcal{H}}}\right)^{-1}=\left(\underline{\underline{C}}^{\ell} \underline{\underline{\mathcal{H}}}^{*}\right)^{-1}$

Virtual changes in the motion parameter vector now become

$\delta \underline{\mathcal{P}}^{*}=\underline{\underline{W}}\left(\underline{\delta \mathcal{U}^{\ell}}-\underline{\delta \mathcal{U}^{k}}\right)$.

Variation in strain energy is expressed as $\delta A=\underline{\mathcal{E}}^{* T} \underline{\mathcal{D}}^{*} \delta \underline{\mathcal{E}}^{*}$ and requires evaluation of the virtual strains, expresse $\overline{\bar{d}}$ as

$\delta \underline{\mathcal{E}}^{*}=\underline{\mathcal{B}}^{*} \delta \underline{\mathcal{P}}^{*}$

where matrix $\underline{B}^{*}$ is defined by Eq. (C1).

Using Eqs. (24) and (23), variation of the strain energy in the flexible joint becomes $\delta A=\left(\underline{\mathcal{U}}^{\ell T}-\delta \underline{\mathcal{U}}^{k T}\right) \underline{W}^{T} \underline{\mathcal{B}}^{T} \underline{\mathcal{L}}^{*}$, where the generalized force vector, $\underline{\mathcal{L}}^{*}$, was defined as

$\underline{\mathcal{L}}^{*}=\underline{\mathcal{D}}^{*} \underline{\mathcal{E}}^{*}$

The elastic forces in the joint now become

$\underline{\mathcal{F}}=\left\{-\frac{\mathcal{F}^{e}}{\underline{\mathcal{F}}^{e}}\right\}$

where

$\underline{\mathcal{F}}^{e}=\underline{\underline{W}}^{T} \underline{\underline{B}}^{* T} \underline{\mathcal{L}}^{*}$ 


\subsection{Stiffness matrix of the flexible joint}

The stiffness matrix of the flexible joint stems from the linearization of the elastic forces defined by Eq. (26). Linearization of $\mathcal{F}^{e}$ yields

$\Delta \underline{\mathcal{F}}^{e}=\Delta \underline{\underline{W}}^{T} \underline{\mathcal{N}}^{*}+\underline{\underline{W}}^{T} \underline{\underline{\mathcal{B}^{*}}} \underline{\underline{\mathcal{L}}}^{*}+\underline{\underline{W}}^{T} \underline{\underline{B}}^{* T} \Delta \underline{\mathcal{L}}^{*}$,

where $\underline{\mathcal{N}}^{*}=\underline{\mathcal{B}}^{* T} \underline{\mathcal{L}}^{*}$. Using Eqs. (24) and (23), the last term of this expression is obtained as $\underline{\underline{W}}^{T} \underline{\mathcal{B}}^{* T} \Delta \underline{\mathcal{L}}^{*}=$ $\left(\underline{\underline{\mathcal{B}}}^{*} \underline{\underline{W}}\right)^{T} \underline{\underline{D^{*}}}\left(\underline{\underline{B}}^{*} \underline{\underline{W}}\right)\left(\underline{\Delta \mathcal{U}^{\ell}}-\underline{\Delta \mathcal{U}^{k}}\right)$.

The first term of Eq. (28) is evaluated next. This term is recast as $\Delta \underline{\underline{W}}^{T} \underline{\mathcal{N}}^{*}=\left[\Delta\left(\underline{\underline{\mathbb{C}}}^{k} \underline{\underline{\mathcal{H}}}\right)^{-T} \underline{\mathcal{N}}^{*}+\Delta\left(\underline{\underline{\mathbb{C}}}^{\ell} \underline{\underline{\mathcal{H}}}^{*}\right)^{-T} \underline{\mathcal{N}}^{*}\right] / 2$ to respect the symmetry of the problem expressed by Eq. (22). Expanding the variations leads to

$$
\Delta \underline{\underline{W}}^{T} \underline{\mathcal{N}}^{*}=\left[-\underline{\underline{W^{T}}} \underline{\underline{\mathcal{T}^{*}}}=\underline{\underline{W}}-\frac{\widehat{\mathcal{F}}^{e}}{2}, \underline{\underline{W^{T}}} \underline{=}=\underline{\underline{\mathcal{T}^{*}}}-\frac{\widehat{\mathcal{F}}^{e}}{2}\right]\left\{\frac{\Delta \underline{\mathcal{U}}^{k}}{\underline{\Delta \mathcal{U}}^{\ell}}\right\}
$$

where notation $(\cdot)$ is defined by Eq. (A5). Matrix $\underline{\mathcal{T}}^{*}=$ $\left[\underline{\underline{\mathcal{H}^{T}}} \underline{\underline{X}}+\underline{\mathcal{H}}^{* T} \underline{\underline{X}} \underline{X}^{*}\right] / 2$, where matrices $\underline{\underline{X}}$ and $\underline{\underline{X}}^{*}$ are implicitly defined by the equations $\Delta \underline{\mathcal{H}}^{-T} \underline{\mathcal{N}}^{*}=\underline{\bar{X}} \Delta \underline{\mathcal{P}}^{*}$ and $\Delta \underline{\mathcal{H}}^{*-T} \underline{\mathcal{N}}^{*}=$ $\underline{X}^{*} \Delta \underline{\mathcal{P}}^{*}$, respectively; explicit expressions of these matrices $\overline{\overline{a r e}}$ given in Eqs. (D4a) and (D4b), respectively.

Finally, the second term of Eq. (28) becomes $\underline{W}^{T} \Delta \underline{\mathcal{B}}^{* T} \underline{\mathcal{L}}^{*}=\underline{\underline{W}}^{T} \underline{Q}^{*}\left(\underline{\mathcal{L}}^{*}, \underline{\mathcal{P}}^{*}\right) \Delta \underline{\mathcal{P}}^{*}$ and Eq. (23) then leads to

$\underline{\underline{\mathcal{W}^{T}}} \Delta \underline{\mathcal{B}}^{* T} \underline{\mathcal{L}}^{*}=\underline{\underline{W}}^{T} \underline{\underline{Q}}^{*}\left(\underline{\mathcal{L}}^{*}, \underline{\mathcal{P}^{*}}\right) \underline{\underline{\underline{W}}}\left(\underline{\delta \mathcal{U}^{\ell}}-\underline{\delta \mathcal{U}^{k}}\right)$.

where the explicit expression of matrix $\underline{Q}^{*}$ is given in Eq. (C2).

The linearized elastic forces can now be written explicitly as

$\Delta \underline{\mathcal{F}}=\underline{\underline{\mathcal{K}}}\left\{\frac{\delta \mathcal{U}^{k}}{\delta \mathcal{U}^{\ell}}\right\}$,

where the stiffness matrix of the elastic joint is

$\underline{\underline{\mathcal{K}}}=\left[\begin{array}{rr}\underline{\underline{D}}+\widehat{\mathcal{F}}^{e} / 2 & -\underline{\underline{D}}+\widehat{\mathcal{F}}^{e} / 2 \\ -\underline{\underline{D}}-\widehat{\mathcal{F}}^{e} / 2 & \underline{\underline{D}}-\widehat{\mathcal{F}}^{e} / 2\end{array}\right]$

where $\underline{\underline{D}}=\underline{\underline{W}}^{T}\left(\underline{\underline{B}}^{* T} \underline{\underline{D}}^{*} \underline{\underline{\mathcal{B}}}+\underline{\mathcal{T}}^{*}+\underline{\underline{Q^{*}}}\right) \underline{\underline{\underline{W}}}$.

\section{Numerical results}

\subsection{Change of reference point}

To illustrate the concepts developed in the previous sections, consider the system consisting of two beams, each of length $L=1.2 \mathrm{~m}$, connected by a flexible joint, as depicted in Fig. 2 . The two beams have the same sectional stiffness properties:

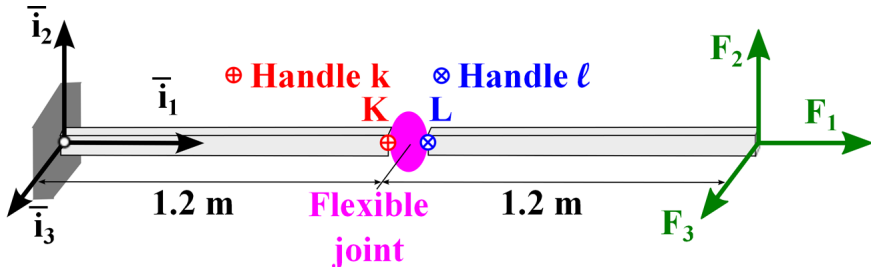

Figure 2. Two beams connected with a flexible joint.

axial stiffness $S=43.50 \mathrm{MN}$; bending stiffness $H_{22}=23.26$ and $H_{33}=298.7 \mathrm{kN} \mathrm{m}^{2}$ about unit vector $\bar{l}_{2}$ and $\bar{l}_{3}$, respectively; torsional stiffness $H_{11}=28.05 \mathrm{kN} \mathrm{m}^{2}$; and shearing stiffness $K_{22}=4.0$ and $K_{33}=2.81 \mathrm{MN}$, along unit vectors $\bar{l}_{2}$ and $\bar{l}_{3}$, respectively.

The first beam is clamped at its root and the second is loaded at its tip by concentrated forces tip loads $F_{1}=$ $500 \alpha / 9, F_{2}=250 \alpha / 9$, and $F_{3}=200 \alpha / 9 \mathrm{~N}$, along unit vectors $\bar{l}_{1}, \bar{l}_{2}$, and $\bar{l}_{3}$, respectively, as shown in Fig. 2 . The load factor $\alpha \in[0,9]$. The stiffness matrix of flexible joint is given by

$$
\underline{\underline{D}}^{*}=\left[\begin{array}{cccccc}
2000 & 10 & 10 & & & \\
10 & 2000 & 10 & & & \\
10 & 10 & 2000 & & & \\
& & & 1000 & & \\
& & & & 1000 & \\
& & & & & 1000
\end{array}\right] .
$$

Two formulations will be contrasted here. In the first, the deformation measures are selected as

$\underline{\mathcal{E}}^{\dagger}=\left\{\begin{array}{l}\underline{\varepsilon}^{\dagger} \\ \underline{\underline{K}}^{\dagger}\end{array}\right\}=\left\{\begin{array}{c}\left(\underline{R}^{k} \underline{\underline{R}}\right)_{0}^{T}\left(\underline{u}^{\ell}-\underline{u}^{k}\right) \\ \operatorname{axial}\left(\underline{\underline{R}}_{0}^{T} \underline{\underline{R}}^{k T} \underline{\underline{R}}^{\ell} \underline{\underline{R}}\right)\end{array}\right\}$.

The stretch vector, $\underline{\varepsilon}^{\dagger}$, corresponds to the components of the relative displacement vector resolved in basis $\mathcal{B}^{k}$. The wryness vector, $\underline{\kappa}^{\dagger}$, is the axial part of the relative rotation tensor, resolved on the same basis; note that the components of the wryness vector selected here are identical when resolved in basis $\mathcal{B}^{k}$ or $\mathcal{B}^{\ell}$.

Various types of deformation measures have been used to represent the behavior of flexible joints, but those given by Eq. (34) are rather typical; in the following sections, they will be referred to as "typical deformation measures". In contrast, the deformation measures used in this work are defined by Eq. (16) and will be referred as "proposed deformation measures".

The main claim of this paper is that the proposed strain measures are invariant with respect to the choice of reference point, i.e., are objective, a characteristic that is not shared by typical deformation measures. Imagine that points $\mathbf{K}$ and $\mathbf{L}$ are interchanged in Fig. 2. Clearly, this does not modify the physical system, and hence, its response under load should be unaffected by this interchange. This basic invariance is satisfied by the proposed deformation measures, but not by their 


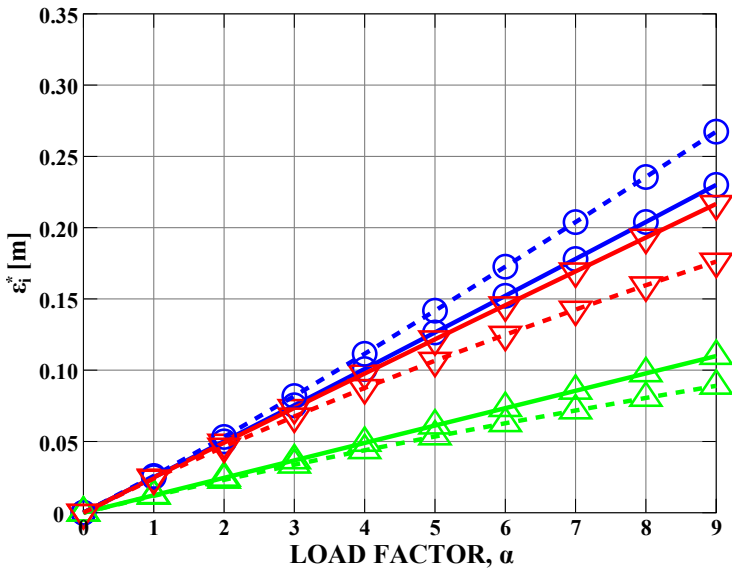

Figure 3. Components of the stretch vector: $\varepsilon_{1}^{*}(\bigcirc), \varepsilon_{2}^{*}(\Delta), \varepsilon_{3}^{*}(\nabla)$. Typical deformation measures. Reference point $\mathbf{K}$ : solid line, point L: dashed line.

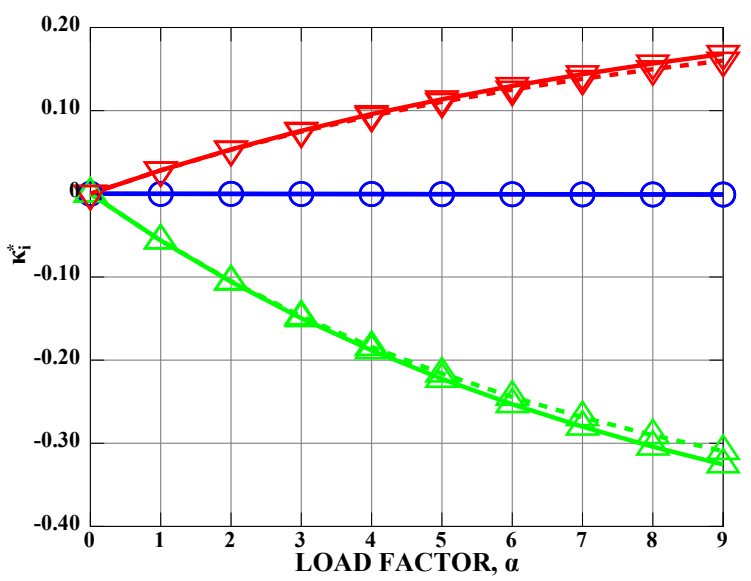

Figure 4. Components of the wryness vector: $\kappa_{1}^{*}(\bigcirc), \kappa_{2}^{*}(\triangle), \kappa_{3}^{*}(\nabla)$. Typical deformation measures. Reference point $\mathbf{K}$ : solid line, point L: dashed line.

typical counterparts. To illustrate this effect, the following parameters were selected for the proposed deformation measures: $\lambda=0$ and $\mu=\left(1-p^{2} / 16\right) /\left(1+p^{2} / 16\right)^{2}$. This choice is not important as the proposed deformation measures are invariant for any choice of these parameters.

For the typical deformation measures, two simulations were performed. In the first run, points $\mathbf{K}$ and $\mathbf{L}$ are selected as indicated in Fig. 2 and in the second simulation, these two points were interchanged. Of course, this interchange is a modeling detail, which has no physical meaning. Yet, these two simulations yield different results because the definition of the deformation measures make specific reference to basis $\mathcal{B}^{k}$, and hence, are inherently "basis sensitive".

Figures 3 and 4 show the components of the stretch and wryness vector components, respectively, versus the load factor, $\alpha \in[0.9]$, for the typical deformation measures defined by Eq. (34). For each deformation component, the response

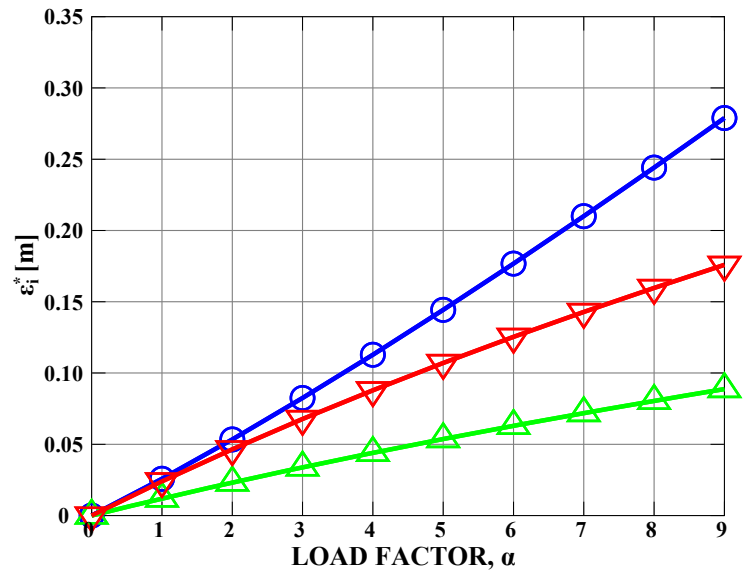

Figure 5. Components of the stretch vector: $\varepsilon_{1}^{*}(\bigcirc), \varepsilon_{2}^{*}(\triangle), \varepsilon_{3}^{*}(\nabla)$. Proposed deformation measures.

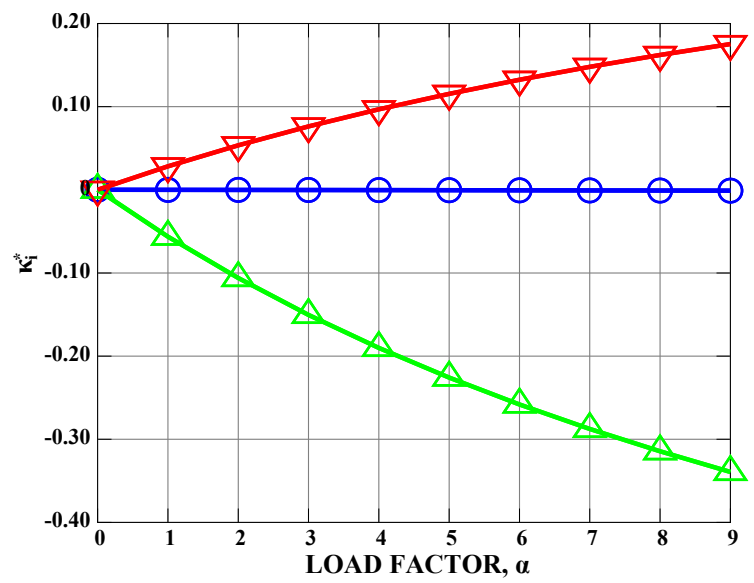

Figure 6. Components of the wryness vector: $\kappa_{1}^{*}(\bigcirc), \kappa_{2}^{*}(\triangle), \kappa_{3}^{*}(\nabla)$. Proposed deformation measures

when reference points $\mathbf{K}$ and $\mathbf{L}$ are selected as shown in Fig. 2 is shown in solid lines, and the response when points $\mathbf{K}$ and $\mathbf{L}$ are interchanged in shown in dashed lines. Clearly, the typical deformation measures do not yield physically meaningful predictions, because different responses are obtained for a given physical system depending on the choice of the labeling convention for points $\mathbf{K}$ and $\mathbf{L}$.

In contrast, the proposed deformation measures yield the same predictions when the roles of points $\mathbf{K}$ and $\mathbf{L}$ are interchanged. Figures 5 and 6 show the components of the stretch and wryness vector components, respectively, for the proposed deformation measures defined by Eq. (16).

Of course, for very small deformations, the predictions based on the typical and proposed deformation measures are identical. This result is expected because both typical and proposed deformation measures converge to the same infinitesimal deformation measures and because identical stiffness matrices were used. 
Table 1. Choices of parameters $\lambda$ and $\mu$ for the sixteen cases.

\begin{tabular}{cccccc}
\hline Case & & $\mathrm{a}$ & $\mathrm{b}$ & $\mathrm{c}$ & $\mathrm{d}$ \\
\hline 1 & $\lambda=0$ & $\mu=1$ & $1+p^{2}$ & $1+p^{4}$ & $1+p^{6}$ \\
2 & $\lambda=0$ & $\mu=1$ & $1+\rho$ & $1+\rho^{2}$ & $1+\rho^{3}$ \\
3 & $\mu=1$ & $\lambda=0$ & $p^{2}$ & $p^{4}$ & $p^{6}$ \\
4 & $\mu=1$ & $\lambda=0$ & $\rho$ & $\rho^{2}$ & $\rho^{3}$ \\
\hline
\end{tabular}

\subsection{Choice of $\lambda$ and $\mu$}

The proposed deformation measures are not unique. Rather, they form families dependent on two arbitrary parameters, $\lambda(\rho, p)$ and $\mu(\rho, p)$ : each choice of these parameters yields a different deformation measure but for all choices, the objectivity and tensorial nature of the deformation measure is preserved. Assuming that the simple strain energy expression defined by Eq. (20) is used with a given stiffness matrix, the choice of parameters $\lambda$ and $\mu$ will alter the response of the flexible joint under load in the nonlinear regime.

To study the influence of the choice of parameters $\lambda$ and $\mu$ on joint behavior, a very simple example was treated. Handle $k$ of the joint was clamped and forces and moments were applied to handle $\ell$; the applied force vector is $\underline{F}^{T}=\left\{\begin{array}{lll}125 & 250 & 375\end{array}\right\} \alpha / 9$ and $\underline{M}^{T}=$ $\left\{\begin{array}{ll}125 & 250 \\ 375\end{array}\right\} \alpha / 9$, where $\alpha$ is the load factor.

A total of sixteen combinations of parameters $\lambda$ and $\mu$ were selected for the study. First, $\lambda=0$ is selected and cases $1 \mathrm{a}$, $1 \mathrm{~b}, 1 \mathrm{c}$, and $1 \mathrm{~d}$ correspond to $\mu=1, \mu=1+p^{2}, \mu=1+p^{4}$, and $\mu=1+p^{6}$, respectively, as listed in the first row of Table 1 . For case 1 , parameter $\mu$ is function of $p$ only. Next, $\lambda=0$ is selected and cases $2 \mathrm{a}, 2 \mathrm{~b}, 2 \mathrm{c}$, and $2 \mathrm{~d}$ correspond to $\mu=1$, $\mu=1+\rho, \mu=1+\rho^{2}$, and $\mu=1+\rho^{3}$, respectively, as listed in the second row of Table 1 . For case 2, parameter $\mu$ is function of $\rho$ only. Cases 3 and 4 are defined similarly, as listed in the third and fourth row of Table 1, respectively.

In all cases, the stiffness matrix of flexible joint was selected as

$$
\stackrel{D}{ }^{*}=\left[\begin{array}{cccccc}
1000 & 100 & 100 & & & \\
100 & 1000 & 100 & & & \\
100 & 100 & 1000 & & & \\
& & & 1000 & & \\
& & & & 1000 & \\
& & & & & 1000
\end{array}\right]
$$

Figures 7 and 8 show the components of the stretch and wryness vectors, respectively, for cases $1 \mathrm{a}, 1 \mathrm{~b}, 1 \mathrm{c}$, and $1 \mathrm{~d}$. The choice of very simple polynomial expressions for $\mu=$ $\mu(p)$ is arbitrary, but all satisfy the limit behavior expressed by Eq. (18). Similarly, the constant value of $\lambda=0$ satisfies the limit behavior expressed by Eq. (19).

For small values of the load factor, the flexible joint deformation remains small and identical response is observed for all choices of parameter $\mu$. As larger loads are applied, the

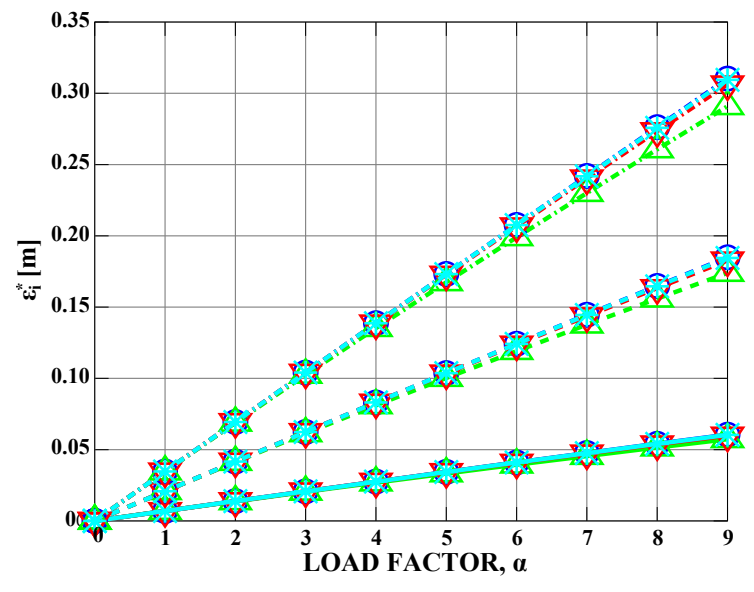

Figure 7. Components of the stretch vector: $\varepsilon_{1}^{*}$ (solid line), $\varepsilon_{2}^{*}$ (dashed line), $\varepsilon_{3}^{*}$ (dash-dotted line). Case 1a $(\bigcirc), 1 \mathrm{~b}(\triangle), 1 \mathrm{c}(\nabla)$, $1 \mathrm{~d}(*)$.

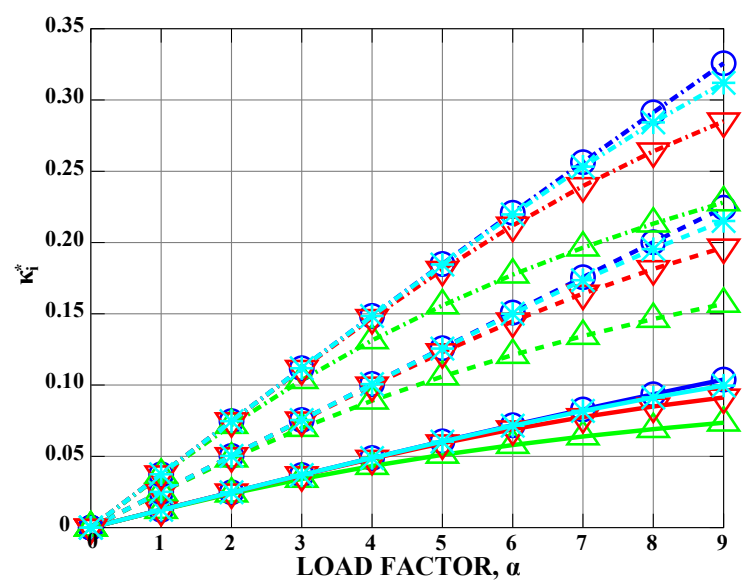

Figure 8. Components of the wryness vector: $\kappa_{1}^{*}$ (solid line), $\kappa_{2}^{*}$ (dashed line), $\kappa_{3}^{*}$ (dash-dotted line). Case 1a $(\bigcirc), 1 \mathrm{~b}(\triangle), 1 \mathrm{c}(\nabla)$, $1 \mathrm{~d}(*)$.

deformation is no longer infinitesimal and the joint's nonlinear response is affected by the choice of parameter $\mu$. This effect is particularly pronounced in the wryness response, as shown in Fig. 8.

Of course, parameters $\lambda$ and $\mu$ are arbitrary functions of both variables $\rho$ and $p$. Case 1 , in which parameter $\mu$ is a function of variable $p$ only, is a special case. In case 2, parameter $\mu$ is selected to be a simple polynomial function of variable $\rho$ only, as shown in the second row of Table 1. Figures 9 and 10 show the components of the stretch and wryness vectors, respectively, for cases $2 \mathrm{a}, 2 \mathrm{~b}, 2 \mathrm{c}$, and $2 \mathrm{~d}$. The nonlinear response of both stretch and wryness vector components is significantly affected by the choice of parameter $\mu$.

Finally, two additional cases, cases 3 and 4, were treated where parameter $\lambda$ is selected to be a function of variables $p$ and $\rho$, respectively, while keeping a constant value of parameter $\mu=1$, as listed in the last two rows of Table 1 , 


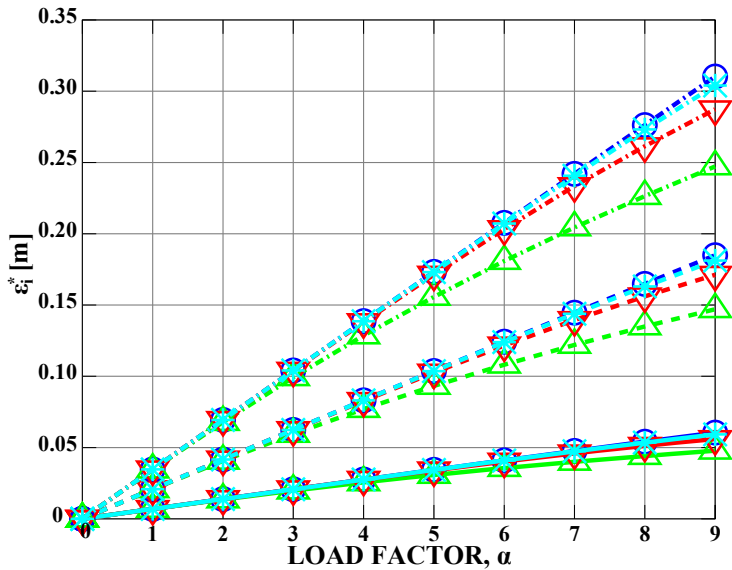

Figure 9. Components of the stretch vector: $\varepsilon_{1}^{*}$ (solid line), $\varepsilon_{2}^{*}$ (dashed line), $\varepsilon_{3}^{*}$ (dash-dotted line). Case 2a $(\bigcirc), 2 \mathrm{~b}(\triangle), 2 \mathrm{c}(\nabla)$, $2 \mathrm{~d}(*)$.

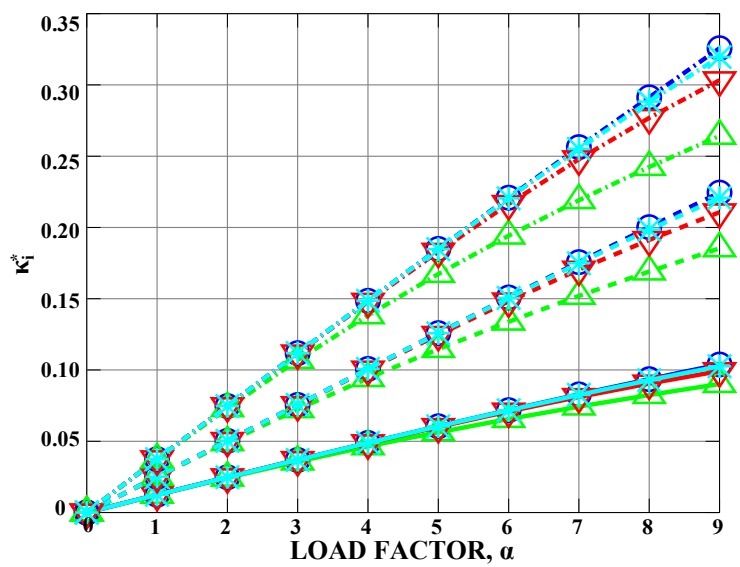

Figure 10. Components of the wryness vector: $\kappa_{1}^{*}$ (solid line), $\kappa_{2}^{*}$ (dashed line), $\kappa_{3}^{*}$ (dash-dotted line). Case 2a $(\bigcirc), 2 \mathrm{~b}(\Delta), 2 \mathrm{c}(\nabla), 2 \mathrm{~d}$ (*).

respectively. Here again, simple polynomial expressions were selected and the limit behaviors expressed by Eqs. (18) and (19) were satisfied. Figures 11 and 12 show the components of the stretch and wryness vectors, respectively, for cases 3a, 3b, 3c, and 3d. Finally, Figs. 13 and 14 show the components of the stretch and wryness vectors, respectively, for cases $4 \mathrm{a}, 4 \mathrm{~b}, 4 \mathrm{c}$, and $4 \mathrm{~d}$.

The goal of the simple examples presented in the previous paragraphs is to show that the nonlinear behavior of the flexible joint is strongly affected by the functional dependency of parameters $\lambda$ and $\mu$ on variables $\rho$ and $p$. Of course, in general, the two parameters can be selected to be functions of both variables, i.e., $\lambda=\lambda(\rho, p)$ and $\mu=\mu(\rho, p)$. Softening or stiffening behavior can be obtained by tailoring the functional dependency of parameters $\lambda$ and $\mu$ on variables $\rho$ and $p$.

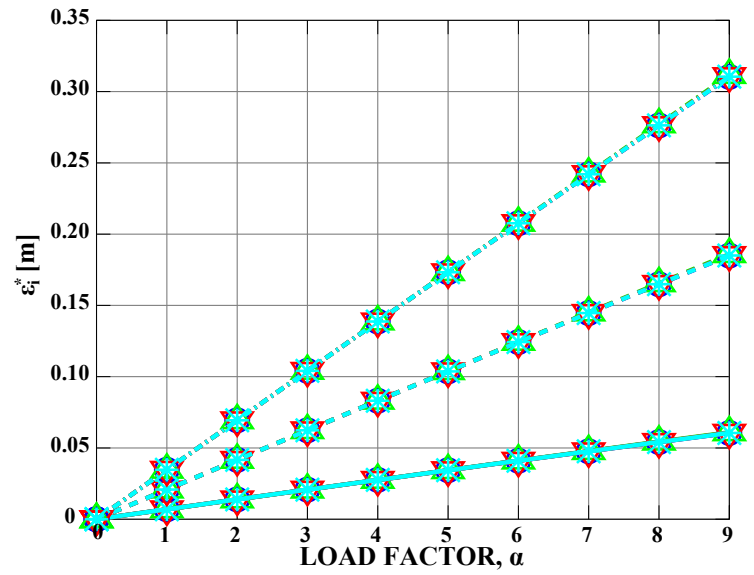

Figure 11. Components of the stretch vector: $\varepsilon_{1}^{*}$ (solid line), $\varepsilon_{2}^{*}$ (dashed line), $\varepsilon_{3}^{*}$ (dash-dotted line). Case 3a $(\bigcirc), 3 \mathrm{~b}(\Delta), 3 \mathrm{c}(\nabla)$, $3 d(*)$.

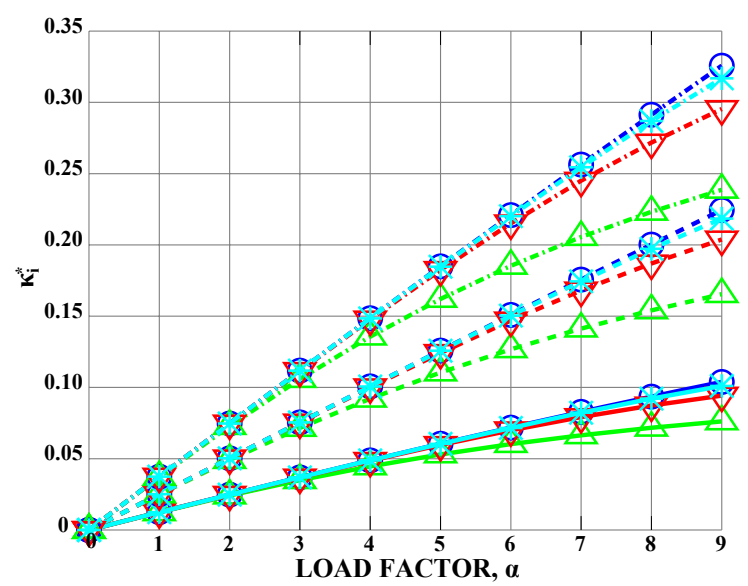

Figure 12. Components of the wryness vector: $\kappa_{1}^{*}$ (solid line), $\kappa_{2}^{*}$ (dashed line), $\kappa_{3}^{*}$ (dash-dotted line). Case 3a $(\bigcirc), 3 \mathrm{~b}(\triangle), 3 \mathrm{c}(\nabla)$, $3 \mathrm{~d}(*)$.

The attention has focused thus far on the strain energy given by Eq. (20), which is a quadratic expression of the deformation measures, $A=\underline{\mathcal{E}}^{* T} \underline{\mathcal{D}}^{*} \underline{\mathcal{E}}^{*} / 2$. This leads to the linear relationship between the generalized forces and proposed deformation measures, $\underline{\mathcal{L}}^{*}=\underline{\mathcal{D}}^{*} \underline{\mathcal{E}}^{*}$, see Eq. (25). This linear relationship, however, is deceptively simple.

Indeed, Eq. (27) shows that the relationship between the elastic forces in the joint and the proposed deformation measures is $\mathcal{F}^{e}=\underline{W}^{T} \underline{\mathcal{B}}^{* T} \mathcal{L}^{*}$. Next, the relationship between the externally applied loads and proposed deformation measures is obtained with the help of Eq. (4) as

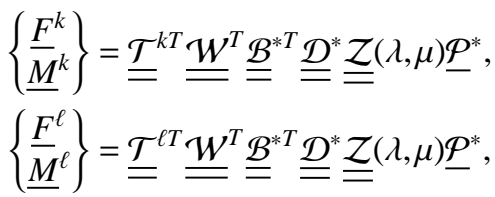




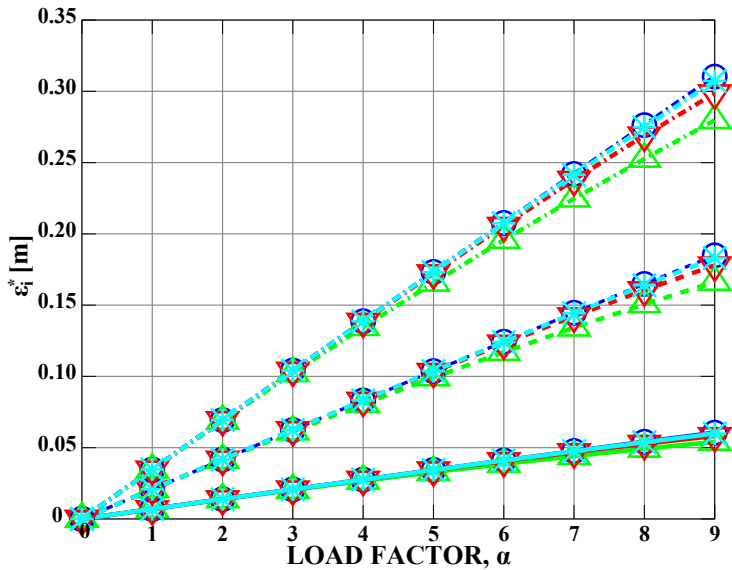

Figure 13. Components of the stretch vector: $\varepsilon_{1}^{*}$ (solid line), $\varepsilon_{2}^{*}$ (dashed line), $\varepsilon_{3}^{*}$ (dash-dotted line). Case 4a $(\bigcirc), 4 \mathrm{~b}(\triangle), 4 \mathrm{c}(\nabla)$, $4 \mathrm{~d}(*)$.

where Eq. (16) was used to express the proposed deformation measures in terms of $\mathcal{P}^{*}$, the relative motion of the joint's two handles. Although the stiffness matrix, $\underline{D}^{*}$, is constant, the relationship between the externally applied loads and the joint's deformation measures is nonlinear because matrices $\underline{\underline{W}}$ and $\underline{\mathcal{B}}^{*}$ are nonlinear functions of the deformations measures, see Eqs. (22) and (C1), respectively, and the relative motion of the two handles, $\mathcal{P}^{*}$, is also a nonlinear function of the handle's relative motion. These observations explain the nonlinear load-deformation behavior exhibited in Figs. 4 to 14 .

In practice, the joint's constitutive laws can be obtained from a two step procedure. First, experimental measurements must be obtained for infinitesimal deformations of the joint and lead to the identification of the entries of the constant stiffness matrix, $\underline{D}^{*}$. Next, experimental measurements characterizing the joint's behavior in the nonlinear range must be obtained. The optimal functional dependencies of parameters $\lambda$ and $\mu$ on variables $\rho$ and $p$ can then be determined using suitable parameter identification methods.

Of course, it is not guaranteed that suitable functions, $\lambda=$ $\lambda(\rho, p)$ and $\mu=\mu(\rho, p)$, can be found, which will model the observed behavior of the joint accurately. In such case, more complex strain energy expressions could be selected in an attempt to better capture the observed constitutive behavior of the joint; in general, $A=A\left(\underline{\mathcal{E}}^{*}\right)$. Of course, the existence of a strain energy function implies that material behavior is conservative, which will not always be true.

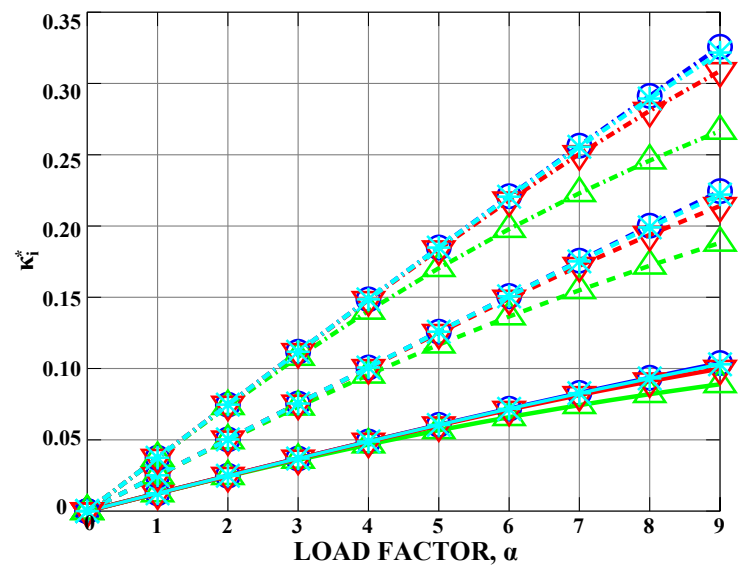

Figure 14. Components of the wryness vector: $\kappa_{1}^{*}$ (solid line), $\kappa_{2}^{*}$ (dashed line), $\kappa_{3}^{*}$ (dash-dotted line). Case 4a $(\bigcirc), 4 \mathrm{~b}(\triangle), 4 \mathrm{c}(\nabla)$, $4 d(*)$.

\section{Conclusions}

This paper has focused on the constitutive behavior of elastic bodies of finite dimension, typically called flexible joints in structural and multibody dynamics. Physically meaningful deformation measures were proposed that are objective and of a tensorial nature; an explicit expression of these measures was derived. Equipped with these deformation measures, constitutive laws for the flexible joint were derived by assuming the existence of a strain energy function that is a quadratic form of these deformation measures. Because all the quantities involved in the formulation are objective and tensorial, the predicted joint behavior presents the required invariance with respect to changes of basis or reference point.

Numerical examples were presented that demonstrate the invariance of the predicted behavior with respect to the choice of reference point, even in the nonlinear range; in contrast, typical formulations found in the literature up to date do not appear to present these desirable characteristics. The proposed deformation measures are not unique: their definition depends on the choice of two parameters, which are functions of the relative rotation and the intrinsic relative displacement at the joint. Numerical examples presented in the paper show that the choice of these two parameters affects the response of the joint in the nonlinear regime significantly. Consequently, the proposed deformation measures form families, and the choice of the functional dependency of the parameter can be selected to tailor the nonlinear response of the joint.

This paper has focused on an expression of the strain energy that depends on the proposed deformation measures in a quadratic manner, leading to a linear relationship between the generalized forces and deformations measure. Despite this linearity, the relationship between externally applied forces and deformations is nonlinear. More general joint constitutive behavior could be obtained by considering more general strain energy expressions. Investigating energy dissipation in 
flexible joint based on the time rate of change of the proposed deformation measure is another possible extension of the this work.

\section{Appendix A}

\section{Notational conventions}

To simplify the writing of this seemingly complicated expression, the following notation is introduced. First, tensor $\underline{Z}$, a function of two scalars, $\alpha$ and $\beta$, is introduced

$\underline{Z}(\alpha, \beta)=\left[\begin{array}{ll}\beta \underline{\underline{I}} & \alpha \underline{\underline{I}} \\ \underline{\underline{0}} & \beta \underline{\underline{I}}\end{array}\right]$.

Second, the generalized vector product tensor is defined

$\widetilde{\mathcal{N}}=\left[\begin{array}{ll}\widetilde{n} & \widetilde{m} \\ \underline{\underline{0}} & \widetilde{n}\end{array}\right]$.

Notation $\widetilde{\mathcal{N}}$ does not indicate a $6 \times 6$ skew-symmetric tensor, but rather the above $6 \times 6$ tensor formed by three skewsymmetric sub-tensors. By analogy to notation $\underline{a}=\operatorname{axial}(\widetilde{a})$, the following operator is introduced

$\underline{\mathcal{N}}=\mathcal{A} x \operatorname{xial}(\widetilde{\mathcal{N}})$.

Consider two vectors defined as

$\underline{\mathcal{V}}=\left\{\begin{array}{l}\underline{v} \\ \underline{\omega}\end{array}\right\}, \quad \underline{\mathcal{P}}=\left\{\frac{p}{q}\right\}$.

The well-known property of the vector product, $\widetilde{a} \underline{b}=-\widetilde{b} \underline{a}$, then generalizes to

$$
\begin{aligned}
\tilde{\mathcal{V}} \underline{\mathcal{P}} & =-\widetilde{\mathcal{P}} \underline{\mathcal{V}}, \\
\widetilde{\mathcal{V}}^{T} \underline{\mathcal{P}} & =\widehat{\mathcal{P}} \underline{\mathcal{V}},
\end{aligned}
$$

where the following notation was introduced

$\widehat{\mathcal{P}}=\left[\begin{array}{ll}\frac{0}{\overline{\widetilde{p}}} & \widetilde{p} \\ \widetilde{q}\end{array}\right]$.

Finally, the following identity results

$\widehat{\widehat{\mathcal{P}} \underline{V}}=\widehat{\mathcal{P}} \widetilde{\mathcal{V}}+\widetilde{\mathcal{V}}^{T} \widehat{\mathcal{P}}$.

\section{Appendix B}

\section{Linearization of functions $\lambda$ and $\mu$}

Scalar $\lambda$ and $\mu$ were introduced in Eq. (17) as functions of $\varrho$ and $p$. Using the chain rule for derivatives, the variation of $\mu$ is $\delta \mu=\mu_{\varrho} \delta \varrho+\mu_{p} \delta p$, where notations $(\cdot)_{\varrho}$ and $(\cdot)_{p}$ indicate derivatives with respect to variable $\varrho$ and angle $p$, respectively. Because $\varrho=\underline{p}^{* T} q^{*}$ and $p^{2}=\underline{p}^{* T} \underline{p}^{*}$, it follows that $\delta \varrho=\underline{p}^{* T} \delta \underline{q}^{*}+\underline{q}^{* T} \delta \underline{p}^{*}$ and $p \delta p=\underline{p}^{* T} \delta \underline{p}^{*}$, and hence,

$\left\{\begin{array}{c}\delta \varrho \\ p \delta p\end{array}\right\}=\left[\begin{array}{ll}\underline{p}^{* T} & \underline{q}^{* T} \\ \underline{0}^{T} & \underline{p}^{* T}\end{array}\right] \delta \underline{\mathcal{P}}^{*}$.

The variations of parameters $\lambda$ and $\mu$ now become

$\left\{\begin{array}{l}\delta \lambda \\ \delta \mu\end{array}\right\}=\underline{\underline{\Lambda}}_{1}(\lambda, \mu)\left[\begin{array}{ll}\underline{p}^{* T} & \underline{q}^{* T} \\ \underline{0}^{T} & \underline{p}^{* T}\end{array}\right] \delta \underline{\mathcal{P}}^{*}$.

where matrix $\underline{\Lambda}_{1}$ is defined as

$\underline{=}_{1}(\lambda, \mu)=\left[\begin{array}{ll}\lambda_{\varrho} & \lambda_{p} / p \\ \mu_{\varrho} & \mu_{p} / p\end{array}\right]$.

Because parameters $\lambda$ and $\mu$ are even functions of variable $p$, their derivatives with respect to the same variable are odd functions of $p$ and hence, $\lambda_{p} / p$ and $\mu_{p} / p$ present no singularity when $p \rightarrow 0$.

Consider two arbitrary arrays, $\underline{\mathcal{L}}^{T}=\left\{\begin{array}{ll}\underline{\ell}^{T} & \underline{m}^{T}\end{array}\right\}$ and $\underline{\mathcal{P}}^{T}=$ $\left\{\begin{array}{ll}q^{T} & \underline{p}^{T}\end{array}\right\}$, and two arbitrary scalars, $\alpha=\alpha(\varrho, p)$ and $\beta=$ $\beta(\varrho, p)$. Matrices $\cong$ and $\varrho$ are defined implicitly by the following two identities, $\bar{\delta} \underline{\underline{Z}}(\alpha, \beta) \underline{\mathcal{L}}=\underline{\underline{Z}}(\underline{\mathcal{L}}, \alpha, \beta, \underline{\mathcal{P}}) \delta \underline{\mathcal{P}}$ and $\delta \underline{\mathcal{Z}}^{T}(\alpha, \beta) \underline{\mathcal{L}}=\underline{\mathcal{Z}}(\underline{\mathcal{L}}, \alpha, \beta, \underline{\mathcal{P}}) \overline{\delta \mathcal{P}}$, respectively. It is shown easily shown that

$\delta \underline{Z}(\alpha, \beta) \underline{\mathcal{L}}=\left[\begin{array}{ll}\frac{m}{0} & \underline{\ell} \\ \underline{m} & \underline{\underline{Z}}\end{array}\right]\left\{\begin{array}{l}\delta \alpha \\ \delta \beta\end{array}\right\}$,

$\delta \underline{\underline{Z}}^{T}(\alpha, \beta) \underline{\mathcal{L}}=\left[\begin{array}{ll}\underline{0} & \underline{\ell} \\ \underline{\underline{m}} & \underline{m}\end{array}\right]\left\{\begin{array}{l}\delta \alpha \\ \delta \beta\end{array}\right\}$.

Introducing Eq. (B2) now yields the following explicit expressions for matrices $\underline{\underline{Z}}$ and $\underline{Z}$,

$\hat{\underline{Z}}(\underline{\mathcal{L}}, \alpha, \beta, \underline{\mathcal{P}})=\left[\begin{array}{ll}\frac{m}{0} & \underline{\ell} \\ \underline{m} & \underline{\underline{\Lambda}}\end{array}\right] \underline{\underline{\Lambda}}_{1}(\alpha, \beta)\left[\begin{array}{ll}\underline{p}^{T} & \underline{q}^{T} \\ \underline{0}^{T} & \underline{p}^{T}\end{array}\right]$,

$\underline{\mathcal{Z}}(\underline{\mathcal{L}}, \alpha, \beta, \underline{\mathcal{P}})=\left[\begin{array}{ll}\underline{0} & \underline{\ell} \\ \underline{m} & \underline{m}\end{array}\right] \underline{\underline{\Lambda}}_{1}(\alpha, \beta)\left[\begin{array}{ll}\underline{p}^{T} & \underline{q}^{T} \\ \underline{\underline{0}}^{T} & \underline{p}^{T}\end{array}\right]$.

\section{Appendix C}

\section{Variations of the strain measures}

The proposed strain measures are defined by Eq. (16) and variations of these quantities are expressed as $\delta \mathcal{E}^{*}=$ $\left[\underline{\underline{Z}}(\lambda, \mu)+\underline{\underline{Z}}\left(\underline{\mathcal{P}}^{*}, \lambda, \mu, \underline{\mathcal{P}}^{*}\right)\right] \delta \underline{\mathcal{P}}^{*}$, where matrix $\underline{\underline{Z}}$ is defined by Eq. (B5a). This implies that $\delta \underline{\mathcal{E}}^{*}=\underline{\mathcal{B}}^{*} \delta \underline{\mathcal{P}}^{*}$, where matrix $\underline{\mathcal{B}}^{*}$ is defined as

$\underline{\underline{B}}^{*}=\underline{\underline{Z}}(\lambda, \mu)+\underline{\underline{Z}}\left(\underline{\mathcal{P}}^{*}, \lambda, \mu, \underline{\mathcal{P}}^{*}\right)$. 
The linearization of the elastic forces requires the evaluation of matrix $\underline{\underline{Q}}$, implicitly defined by $\delta \underline{\mathcal{B}}^{*} \underline{\underline{L}}^{*}=\underline{\underline{Q}}^{*} \delta \underline{\mathcal{P}}^{*}$. The previous results yield

$$
\begin{aligned}
\underline{\underline{Q}}^{*}= & \underline{Z}(\epsilon, \gamma)+\underline{\underline{Z}}\left(\underline{\mathcal{P}}^{*}, \lambda, \mu, \underline{\mathcal{L}}^{*}\right)+\underline{\underline{Z}}\left(\underline{\mathcal{L}}^{*}, \lambda, \mu, \underline{\mathcal{P}}^{*}\right) \\
& +\left[\begin{array}{ll}
\underline{p}^{*} & \underline{q}^{*} \\
\underline{\underline{p}} & \underline{\underline{p}}^{*}
\end{array}\right] \underline{\underline{\mathcal{S}}}\left[\begin{array}{ll}
\underline{p}^{* T} & \underline{q}^{* T} \\
\underline{p}^{* T} & \underline{\underline{x}}^{T}
\end{array}\right],
\end{aligned}
$$

where the following scalar functions were defined

$\left\{\begin{array}{l}\alpha \\ \beta\end{array}\right\}=\left[\begin{array}{ll}\underline{p}^{* T} & \underline{q}^{* T} \\ \underline{0}^{T} & \underline{p}^{* T}\end{array}\right] \underline{\mathcal{L}}^{*},\left\{\begin{array}{l}\epsilon \\ \gamma\end{array}\right\}=\left[\begin{array}{ll}\lambda_{\varrho} & \lambda_{p} / p \\ \mu_{\varrho} & \mu_{p} / p\end{array}\right]\left\{\begin{array}{l}\alpha \\ \beta\end{array}\right\}$,

and

$\underline{\mathcal{S}}=\left[\begin{array}{ll}\alpha \lambda_{\varrho \varrho}+\beta \lambda_{\varrho p} / p & \alpha \lambda_{\varrho p} / p+\beta\left(\lambda_{p p}-\lambda_{p} / p\right) / p^{2} \\ \alpha \mu_{\varrho \varrho}+\beta \mu_{\varrho p} / p & \alpha \mu_{\varrho p} / p+\beta\left(\mu_{p p}-\mu_{p} / p\right) / p^{2}\end{array}\right]$.

\section{Appendix D}

\section{Linearization of the tangent tensor}

The linearization of the elastic forces also requires linearization of the tangent tensor. When using the WienerMilenković motion parameterization, the tangent tensor is expressed as

$$
\begin{aligned}
& \underline{\underline{\mathcal{H}}}^{-1}(\underline{\mathcal{P}})=\underline{Z}\left(\bar{\chi}_{0}, \chi_{0}\right)-\widetilde{\mathcal{P}} / 2+\underline{Z}\left(\bar{\chi}_{2}, \chi_{2}\right) \widetilde{\mathcal{P}} \widetilde{\mathcal{P}}, \\
& \underline{\underline{\mathcal{H}}}^{*-1}(\underline{\mathcal{P}})=\underline{\underline{Z}}\left(\bar{\chi}_{0}, \chi_{0}\right)+\widetilde{\mathcal{P}} / 2+\underline{Z}\left(\bar{\chi}_{2}, \chi_{2}\right) \widetilde{\mathcal{P}} \widetilde{\mathcal{P}} .
\end{aligned}
$$

The linearization of the tangent tensor is achieved by defining matrices $\underline{X}$ and $\underline{X}^{*}$ implicitly defined by the following expressions, $\Delta \underline{\mathcal{H}}^{-T} \underline{\underline{\mathcal{L}}}=\underline{\underline{X}}(\underline{\mathcal{L}}) \Delta \underline{\mathcal{P}}$ and $\underline{\mathcal{H}}^{*-T} \underline{\mathcal{L}}=\underline{\mathcal{X}}^{*}(\underline{\mathcal{L}} \Delta \underline{\mathcal{P}}$. Tedious algebra yields explicit equations of these matrices as

$$
\begin{aligned}
& \underline{\underline{X}}(\underline{\mathcal{L}})=\underline{\underline{Z}}\left(\underline{\mathcal{L}}, \bar{\chi}_{0}, \chi_{0}, \underline{\mathcal{P}}\right)+\underline{\underline{Z}}\left(\widehat{\mathcal{N}} \underline{\mathcal{P}}, \bar{\chi}_{2}, \chi_{2}, \underline{\mathcal{P}}\right) \\
& -\widehat{\mathcal{L}} / 2+\underline{Z}^{T}\left(\bar{\chi}_{2}, \chi_{2}\right)\left(\widehat{\mathcal{N}}+\widetilde{\mathcal{P}}^{T} \widehat{\mathcal{L}}\right), \\
& \underline{\underline{X}}^{*}(\underline{\mathcal{L}})=\underline{\underline{Z}}\left(\underline{\mathcal{L}}, \bar{\chi}_{0}, \chi_{0}, \underline{\mathcal{P}}\right)+\underline{\underline{Z}}\left(\widehat{\mathcal{N}} \underline{\mathcal{P}}, \bar{\chi}_{2}, \chi_{2}, \underline{\mathcal{P}}\right) \\
& +\widehat{\mathcal{L}} / 2+\underline{\mathcal{Z}}^{T}\left(\bar{\chi}_{2}, \chi_{2}\right)\left(\widehat{\mathcal{N}}+\widetilde{\mathcal{P}}^{T} \widehat{\mathcal{L}}\right),
\end{aligned}
$$

where $\underline{\mathcal{N}}=\widetilde{\mathcal{P}}^{T} \underline{\mathcal{L}}=\widehat{\mathcal{L}} \underline{\mathcal{P}}$ and matrix $\underline{\check{Z}}$ is defined by Eq. (B5b).

The Wiener-Milenković rotation parameterization is defined as $p=p(\phi) \bar{n}$, where $p(\phi)=4 \tan \phi / 4$. Table D1 lists the expressions for all the scalar functions appearing in the above expression when the Wiener-Milenković motion parameterization is used.

Matrices $\underline{\mathcal{X}}$ and $\underline{\mathcal{X}}^{*}$ defined in Eqs. (D2a) and (D2b),

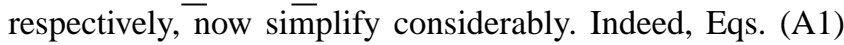
and (B5b) imply $\underline{Z}^{T}\left(\bar{\chi}_{2}, \chi_{2}\right)=\underline{\underline{I}} / 8, \underline{\underline{\mathcal{Z}}}\left(\widehat{\mathcal{N}} \underline{\mathcal{P}}, \bar{\chi}_{2}, \chi_{2}, \underline{\mathcal{P}}\right)=\underline{\underline{0}}$, and

$\underline{\underline{Z}}\left(\underline{\mathcal{L}}, \bar{\chi}_{0}, \chi_{0}, \underline{\mathcal{P}}\right)=\frac{1}{8}\left[\begin{array}{ll}\underline{0} & \underline{\ell} \\ \underline{\underline{\ell}} & \underline{\underline{\ell}}\end{array}\right]\left[\begin{array}{ll}\underline{p}^{T} & \underline{q}^{T} \\ \underline{0}^{T} & \underline{p}^{T}\end{array}\right]$,
Table D1. Scalars functions associated with the Wiener-Milenković motion parameterization.

\begin{tabular}{cl|rl}
\hline Quantity & Value & Quantity & Value \\
\hline$v$ & $1 /\left(1+p^{2} / 16\right)$ & $\varepsilon$ & $1 /\left(1-p^{2} / 16\right)$ \\
$p^{\prime}$ & $1+p^{2} / 16$ & $\bar{\varepsilon}$ & $\varrho \varepsilon^{2} / 8$ \\
$\zeta_{1}$ & $v^{2} / \varepsilon$ & $\zeta_{2}$ & $v^{2} / 2$ \\
$\bar{\zeta}_{1}$ & $\varrho v^{2}(1-4 v) / 8$ & $\bar{\zeta}_{2}$ & $-\varrho v^{3} / 8$ \\
$\sigma_{0}$ & $v$ & $\sigma_{2}$ & $v^{2} / 8$ \\
$\bar{\sigma}_{0}$ & $-\varrho v^{2} / 8$ & $\bar{\sigma}_{2}$ & $-\varrho v^{3} / 32$ \\
$\chi_{0}$ & $1 / v$ & $\chi_{2}$ & $1 / 8$ \\
$\bar{\chi}_{0}$ & $\varrho / 8$ & $\bar{\chi}_{2}$ & 0 \\
\hline
\end{tabular}

and finally,

$$
\begin{aligned}
& \underline{X}(\underline{\mathcal{L}})=\underline{\underline{Z}}\left(\underline{\mathcal{L}}, \bar{\chi}_{0}, \chi_{0}, \underline{\mathcal{P}}\right)-\widehat{\mathcal{L}} / 2+\left(\widehat{\mathcal{N}}+\widetilde{\mathcal{P}}^{T} \widehat{\mathcal{L}}\right) / 8, \\
& \underline{\underline{\mathcal{X}^{*}}}(\underline{\mathcal{L}})=\underline{\underline{Z}}\left(\underline{\mathcal{L}}, \bar{\chi}_{0}, \chi_{0}, \underline{\mathcal{P}}\right)+\widehat{\mathcal{L}} / 2+\left(\widehat{\mathcal{N}}+\widetilde{\mathcal{P}}^{T} \widehat{\mathcal{L}}\right) / 8 .
\end{aligned}
$$

Acknowledgements. This work was not sponsored by any agency as I was transferring from the USA to China and was between the two systems.

Edited by: A. Tasora

Reviewed by: two anonymous referees

\section{References}

Anand, L.: On H. Hencky's approximate strain-energy function for moderate deformations, J. Appl. Mech., 46, 78-82, 1979.

Anand, L.: Moderate deformations in extension-torsion of incompressible isotropic elastic materials, J. Mech. Phys. Solids, 34, 293-304, 1986.

Angeles, J.: On twist and wrench generators and annihilators, in: Computer-Aided Analysis Of Rigid And Flexible Mechanical Systems, edited by: Pereira, M. S. and Ambrosio, J., Dordrecht, NATO ASI Series, Kluwer Academic Publishers, 379-411, 1993.

Argyris, J.: An excursion into large rotations, Comput. Method. Appl. M., 32, 85-155, 1982.

Borri, M., Trainelli, L., and Bottasso, C.: On representations and parameterizations of motion, Multibody Syst. Dyn., 4, 129-193, 2000.

Bauchau, O.: Flexible Multibody Dynamics, Dordrecht, Heidelberg, London, New-York, Springer, 2011.

Bauchau, O. and Choi, J.: The vector parameterization of motion, Nonlinear Dynam., 33, 165-188, 2003.

Bauchau, O. and Craig, J.: Structural Analysis with Application to Aerospace Structures, Springer, Dordrecht, Heidelberg, London, New-York, 2009.

Bauchau, O. and Li, L.: Tensorial parameterization of rotation and motion, J. Comput. Nonlin. Dyn., 6, 1-8, 2011.

Bauchau, O. and Trainelli, L.: The vectorial parameterization of rotation, Nonlinear Dynam., 32, 71-92, 2003.

Degener, M., Hodges, D. H., and Petersen, D.: Analytical and experimental study of beam torsional stiffness with large axial elongation, J. Appl. Mech., 55, 171-178, 1988. 
Ibrahimbegović, A.: On the choice of finite rotation parameters, Comput. Method. Appl. M., 149, 49-71, 1997.

Kadlowec, J., Wineman, A., and Hulbert. G.: Elastomer bushing response: Experiments and finite element modeling, Acta Mech., 163, 25-38, 2003.

Kane, T.: Dynamics, New York, Holt, Rinehart and Winston, Inc., 1968.

Ledesma, R., Ma, Z.-D., Hulbert, G., and Wineman, A.: A nonlinear viscoelastic bushing element in multibody dynamics, Comput. Mech., 17, 287-296, 1996.

Malvern, L. E.: Introduction to the Mechanics of a Continuous Medium, Prentice Hall, Inc., Englewood Cliffs, New Jersey.

Masarati, P. and Morandini, M.: Intrinsic deformable joints, Multibody Syst. Dyn., 23, 361-386, 2010.

Merlini, T. and Morandini, M.: The helicoidal modeling in computational finite elasticity. Part I: Variational formulation, Int. J. Solids Struct., 41, 5351-5381, 2004.
Milenković, V.: Coordinates suitable for angular motion synthesis in robots, in: Proceedings of the Robot VI Conference, Detroit MI, 2-4 March, Paper MS82-217, 1982.

Pennestrì, E. and Stefanelli, R.: Linear algebra and numerical algorithms using dual numbers, Multibody Syst. Dyn., 18, 323-344, 2007.

Shuster, M. A survey of attitude representations, J. Astronaut. Sci., 41, 439-517, 1993.

Wiener, T.: Theoretical Analysis of Gimballess Inertial Reference Equipment Using Delta-Modulated Instruments, Ph.D. thesis, Massachusetts Institute of Technology, Department of Aeronautical and Astronautical Engineering, Cambridge, Massachusetts, 1962. 